\title{
Osteomielitis multifocal por Serratia marcescens en un niño con enfermedad granulomatosa crónica
}

\section{Serratia marcescens multifocal osteomyelitis in a child with chronic granulomatous disease}

María Eugenia Castro-Moraga', Paulina Coria ${ }^{2,3}$, Natalia Conca ${ }^{2,3}$, Javiera Berho ${ }^{2,4}$ y Alejandra King ${ }^{2,4}$

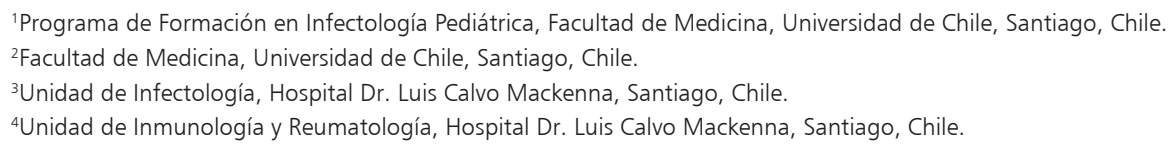

Los autores declaran no tener conflicto de interés.

Recibido: 11 de enero de 2021 / Aceptado: 16 de julio de 2021

\section{Resumen}

La enfermedad granulomatosa crónica (EGC) es una inmunodeficiencia primaria poco frecuente. Se caracteriza por una alteración en la función de los fagocitos, causando infecciones recurrentes bacterianas y fúngicas. Presentamos el caso clínico de un niño con una osteomielitis multifocal por Serratia marcescens, microorganismo infrecuente como causa de infecciones óseas en niños, aunque asociado a la EGC. El estudio de infecciones con presentación clínica y agentes inhabituales deben hacer sospechar una EGC. Su diagnóstico precoz en la vida, así como el tratamiento antimicrobiano oportuno y el uso posterior de una profilaxis antimicrobiana adecuada logrará evitar recurrencias infecciosas y secuelas.

Palabras clave: Enfermedad granulomatosa crónica; inmunodeficiencia primaria; osteomielitis; Serratia marcescens.

\section{Introducción}

$\mathrm{L}$ a enfermedad granulomatosa crónica (EGC) es una afección monogenética causada por defectos en el complejo enzimático nicotinamida-adenina-dinucleótido-fosfato (NADPH)-oxidasa de los macrófagos, cuya función es generar compuestos reactivos derivados del oxígeno, fundamentales en la lisis de los microorganismos fagocitados. Esta anomalía predispone el desarrollo de infecciones bacterianas y fúngicas recurrentes, potencialmente mortales, con la formación de granulomas.

\section{Abstract}

Chronic granulomatous disease (CGD) is a rare primary immunodeficiency. It is characterized by an alteration in the function of phagocytes causing recurrent bacterial and fungal infections. This is a case report of a child with multifocal osteomyelitis by Serratia marcescens, an infrequent as a cause of bone infections, although associated with CGD. The study of infections with clinical presentation and unusual agents should lead to suspicion of CGD. The diagnosis early in life, as well as timely antimicrobial treatment and the subsequent antimicrobial prophylaxis will avoid infectious recurrences and sequelae.

Key words: Chronic granulomatous disease; primary immunodeficiency; osteomyelitis, Serratia marcescens.

\section{Correspondencia a:}

María Eugenia Castro-Moraga.

castro.maeugenia@gmail.com

La EGC constituye una inmunodeficiencia primaria poco frecuente con incidencias descritas de 1/200.000 a 1/70.000 recién nacidos vivos, dependiendo de la población estudiada ${ }^{1,2}$. Afecta con mayor frecuencia a varones en una relación aproximada de 2:1, esto debido a que 65$70 \%$ de las mutaciones están ligadas al cromosoma X. Las formas con herencia autosómica recesiva se describen con mayor frecuencia en comunidades con alta consanguineidad ${ }^{2,3}$.

La EGC es causada por mutaciones que resultan en la pérdida o inactivación funcional de alguna de las seis proteínas necesarias para formar el complejo NADPH-oxidasa. En estado basal, existe 
como dos componentes: el primero, un heterodímero unido a la membrana citoplasmática del fagocito llamado citocromo b-245, que se compone de las glicoproteínas gp91-phox y p22-phox; y tres proteínas presentes en el citosol (p47-phox, p67-phox y p40-phox). El ensamblaje ocurre en presencia de la proteína esencial para las especies reactivas de oxígeno (EROS) ${ }^{4,5}$. Una vez que se ha formado el complejo NADPH-oxidasa, se toma un electrón de NADPH y se dona al oxígeno molecular, lo que lleva a la formación de superóxido. Este se convierte en peróxido de hidrógeno, el que finalmente, reacciona con el anión superóxido, formando un radical hidroxilo altamente reactivo que se convierte en ácido hipocloroso en presencia de mieloperoxidasa y cloro en el fagosoma de los neutrófilos ${ }^{4,6}$. La producción de especies reactivas de oxígeno (ROS) por parte de los fagocitos conduce a la entrada de potasio y protones al fagolisosoma, lo que lleva a la activación de proteasas granulares, incluidas elastasa y catepsina G. Estas proteasas son responsables de la destrucción de microorganismos fagocitados ${ }^{7}$. Por lo tanto, el superóxido actúa como microbicida directo y como una molécula de activación intracelular. Finalmente, resulta en la incapacidad de los neutrófilos, monocitos y macrófagos para destruir ciertos microorganismos predisponiendo el desarrollo de infecciones ${ }^{1}$.

Del punto de vista microbiológico, los principales agentes que infectan a los pacientes con EGC son productores de catalasa. La catalasa es una enzima "antioxidante" que inactiva el peróxido de hidrógeno que normalmente producen algunas bacterias y hongos durante su crecimiento ${ }^{1}$.

Los principales sitios de infección en pacientes con EGC son pulmón, piel, ganglios linfáticos, hígado y hueso ${ }^{8,9}$. Los agentes etiológicos más frecuentemente descritos son: Staphylococcus aureus, Burkholderia cepacia complex, Nocardia spp., Serratia marcescens y Aspergillus spp. En algunos países también se describen Salmonella spp., bacilo Calmette-Guerin (BCG) y Mycobacterium tuberculosis ${ }^{10}$.

Se presenta el caso de una osteomielitis multifocal por Serratia marcescens, microorganismo poco frecuente como agente etiológico de osteomielitis en la infancia, lo que orientó a la sospecha diagnóstica de esta inmunodeficiencia primaria.

\section{Caso clínico}

Preescolar de 3 años 4 meses, sexo masculino, con antecedente de una infección urinaria febril a los tres meses de vida, y dos episodios de neumonía, ambas con hospitalizaciones prolongadas y uso de antimicrobianos intravenosos, sin aislamiento microbiológico. Nacido en Perú, hace un año había migrado a Chile con su familia.
Tenía sus vacunas al día, habiendo recibido en su país de origen, BCG y VHB al nacer, el esquema completo de vacunas pentavalente, polio, rotavirus, neumocócica $13 \mathrm{~V}$ y dos dosis de tresvírica. Las inmunizaciones del PNI chileno contra VHA y meningocócica ACWY se encontraban pendientes. No tenía antecedentes familiares de enfermedades de importancia. Sus padres eran padres consanguíneos en grado no precisado y tenía dos hermanos sanos, una niña y un varón, este último de distinto padre.

Consultó en su hospital de origen por dolor y aumento de volumen del codo izquierdo días después de una caída, mientras jugaba. Evolucionó con eritema y mayor aumento de volumen local. En la evaluación clínica se encontraba decaído, pálido, afebril y normotenso. Al examen físico presentaba un aumento de volumen, calor local y dolor en el codo izquierdo. Dentro de sus exámenes iniciales destacaban una anemia con hematocrito de 27,1\%, elevación de PCR a $69 \mathrm{mg} / \mathrm{l}$ y de la velocidad de sedimentación a $53 \mathrm{~mm} / \mathrm{h}$, sin leucocitosis. La radiografía del codo izquierdo mostró una reacción perióstica con aumento de volumen de tejidos blandos. Se realizó una exploración quirúrgica que evidenció una lesión ósea macroscópica compatible con una osteomielitis, realizándose el drenaje de un contenido hemopurulento. Se inició antibioticoterapia empírica con penicilina sódica 300.000 $\mathrm{UI} / \mathrm{kg} /$ día y clindamicina $30 \mathrm{mg} / \mathrm{kg} /$ día. El resultado de cultivo óseo informó desarrollo de Serratia marcescens, identificado por sistema Vitek ${ }^{\circledR}$ 2. El antibiograma mostró sensibilidad a amikacina, ciprofloxacina, ceftriaxona, cefotaxima, gentamicina y cotrimoxazol; por lo que se agregó amikacina $15 \mathrm{mg} / \mathrm{kg} /$ día.

Al quinto día postoperatorio se pesquisó un aumento de volumen redondeado de aproximadamente $2 \mathrm{~cm}$, esta vez del codo derecho, observándose en la radiografía un engrosamiento del periostio y lesión osteolítica en el olécranon. Debido a la evolución tórpida, se derivó a nuestro hospital con el diagnóstico de osteomielitis en ambos codos, para completar el estudio y terapia.

A su ingreso se ajustó el esquema antibacteriano a ciprofloxacina intravenosa $30 / \mathrm{mg} / \mathrm{kg} /$ día para el tratamiento del primer agente aislado y cloxacilina $100 \mathrm{mg} / \mathrm{kg} /$ día, para cubrir agentes habituales ante la nueva lesión. Los hemocultivos fueron negativos. Las radiografías de control informaron una lesión lítica con reacción perióstica densa en el húmero distal izquierdo y a nivel de ulna proximal derecha (Figura 1). Se realizó una nueva intervención quirúrgica, esta vez de ambos codos, consistentes en drenaje y toma de biopsia y cultivo. El estudio histopatológico de húmero izquierdo informó tejido fibro-colagenoso vascularizado y muscular estriado sin hallazgos significativos y, la ulna derecha se describió como un fragmento de tejido óseo trabecular con espacios intertrabeculares con un mode- 

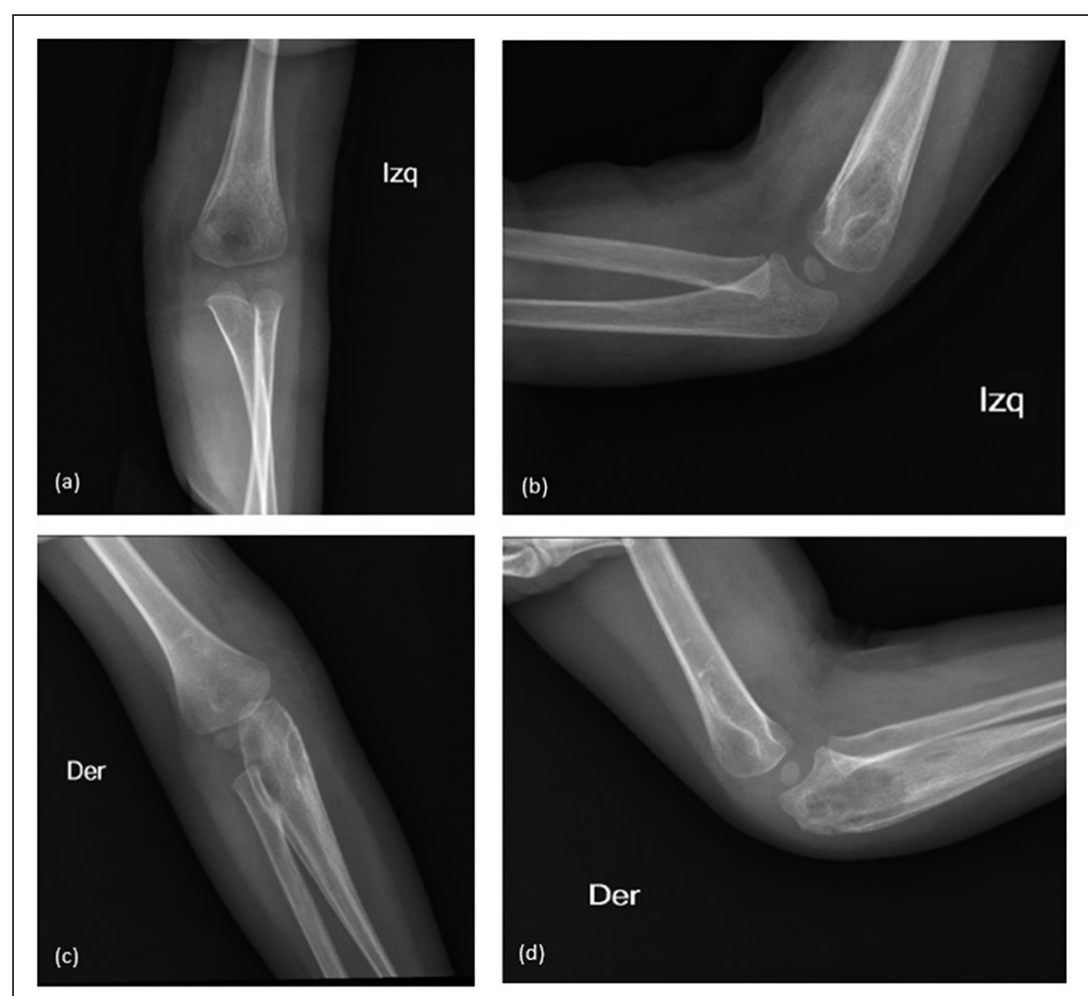

Figura 1. Radiografías con proyección anteroposterior (a) y lateral (b) del codo izquierdo: lesión lítica con reacción perióstica densa a nivel de húmero distal izquierdo; radiografías con proyección anteroposterior (c) y lateral (d) de codo derecho: lesión lítica con reacción perióstica densa a nivel de la ulna proximal derecha. cirugía. La serología de VIH fue no reactiva; el estudio de inmunoglobulinas mostró una hipergamaglobulinemia policlonal, con IgG $3.460 \mathrm{mg} / \mathrm{dl}$, IgA $247 \mathrm{mg} / \mathrm{dl}$ e IgM $174 \mathrm{mg} / \mathrm{dl}$; subpoblaciones linfocitarias y complemento C3 y C4 normales; y anti-toxoide tetánico 0,5 UI/ml (valor de referencia $>0,15 \mathrm{UI} / \mathrm{ml}$ ).

Ya que se trataba de una osteomielitis bilateral, de etiología y evolución no habitual, se realizó la prueba de dihidrorodamina (DHR) en tres ocasiones para descartar una EGC. Las primeras dos tuvieron resultado indeterminado y el tercer examen mostró un índice de estimulación de 0,6 (anormal < 30; EGC < 5). Se diagnosticó así una EGC, iniciando profilaxis con cotrimoxazol $5 \mathrm{mg} / \mathrm{kg} /$ día de trimetoprim en forma diaria, para cobertura de Nocardia spp., S. aureus y bacilos gramnegativos y, profilaxis antifúngica para hongos filamentosos con voriconazol $15 \mathrm{mg} / \mathrm{kg} /$ día.

El paciente evolucionó satisfactoriamente. Recibió tres semanas de ciprofloxacina i.v y luego oral, completando seis semanas de terapia. Se hizo seguimiento en el policlínico de especialidad al alta, sin presentar nuevas infecciones. Desde el punto de vista inmunológico, se repitió DHR a los seis meses y nuevamente resultó alterado, con un índice de estimulación de 3,81 compatible con EGC. El estudio genético no se ha podido realizar hasta la fecha, ya que el paciente regresó a su ciudad de residencia.

\section{Discusión}

En el caso presentado, el paciente tenía antecedente de neumonías a repetición, pero fue la infección ósea bilateral por $S$. marcescens la que levantó la sospecha inicial de EGC. Galluzzo y cols. describieron en una cohorte de 20 años, una incidencia de osteomielitis de un 30\% en pacientes con EGC, siendo la primera manifestación de la enfermedad en $17 \%$ de los casos $^{9}$. A pesar que la osteomielitis por Serratia spp. se describe en pacientes con EGC entre un 8 y $13 \%{ }^{11,12}$ y hasta $50 \%$ como agente etiológico ${ }^{9}$, no es un patógeno reportado en nuestro medio de infecciones en niños con EGC. Si bien se presenta con mayor frecuencia en la edad preescolar ${ }^{9}$, también hay casos descritos en recién nacidos ${ }^{13}$. En la niñez la mayoría de los casos ocurre en los huesos largos, con solo 1 a 4\% en las manos o los pies.

La tasa de recurrencia de infección por Serratia se ha descrito en $18 \%$, con afectación de diversos sitios, inclusive distintos al foco inicial ${ }^{11}$, por lo que el tiempo de tratamiento y seguimiento es muy importante. En el caso de nuestro paciente, se trató por seis semanas con ciprofloxacina, sin recurrencias, con un estudio electrocardiográfico intratratamiento normal. Ciprofloxacina, al ser una molécula lipofílica con alto volumen de 
distribución y mayor relación en la concentración hueso/suero respecto de otros antimicrobianos, logra una mejor penetrancia a la matriz ósea en comparación con amikacina, prefiriéndose como terapia. Si bien, el rango de tiempo de tratamiento para una osteomielitis crónica se recomienda entre 6 a 12 semanas, en este caso dada la mejoría clínica y la posibilidad de contar con un control ambulatorio precoz, fue posible suspender el tratamiento, sin evidencia de reinfección en los controles posteriores.

La osteomielitis en la infancia, particularmente cuando es causada por agentes inhabituales como $S$. marcescens, debe hacer sospechar una $\mathrm{EGC}^{14}$. Sin embargo, es importante destacar que ante una evolución no habitual o recurrente de infecciones óseas por microorganismos típicos para la edad (como S. aureus o Kingella kingae), también debe descartarse una EGC. En general, Serratia spp. tiene una baja virulencia y se considera un patógeno oportunista, proveniente de fuentes ambientales más que de la microbiota, siendo frecuente como agente causal de infecciones y brotes nosocomiales ${ }^{15}$. Sin embargo, las infecciones por $S$. marcescens en pacientes con EGC adquiere una mayor relevancia, con una incidencia descrita cercana a 1 caso por 100 pacientes por año ${ }^{11}$.

Las infecciones por micobacterias también se han asociado a EGC, sobre todo en pacientes que viven en áreas endémicas de tuberculosis, por lo que en nuestro paciente se realizó una búsqueda dirigida de este microorganismo. Una revisión de casos de infección por micobacterias en pacientes con EGC durante 35 años reportó un total de 72 pacientes; de ellos $53 \%$ presentaron enfermedad por vacuna $\mathrm{BCG}, 6 \%$ enfermedad atribuida a micobacterias medioambientales, $22 \%$ tuvo diagnóstico de tuberculosis, $10 \%$ de ellos con tuberculosis y enfermedad por BCG y $10 \%$ enfermedad causada por Mycobacterium spp. Este estudio enfatiza que los pacientes con enfermedad clínica grave causada por BCG y/o M. tuberculosis deben ser investigados no solo para inmunodeficiencia primaria combinada severa o defectos en el circuito IL-12/23IFN- $\gamma$, sino también para $\mathrm{EGC}^{16}$.

Además de la sospecha clínica, el diagnóstico de EGC se realiza con técnicas de evaluación de la función fagocítica, y se confirma encontrando el defecto molecular exacto mediante genotipificación. Dentro de las técnicas de evaluación de la fagocitosis la más usada en nuestro medio es la prueba de dihidrorodamina (DHR) por citometría de flujo, más conocido como "estallido respiratorio", que evalúa la capacidad de los neutrófilos de producir ROS, reflejando el rápido consumo de oxígeno y la producción de superóxido y sus metabolitos ${ }^{4,6}$.

Este caso clínico demuestra la importancia de la historia clínica como un elemento clave para la sospecha de inmunodeficiencias. De acuerdo con las banderas rojas establecidas por Jeffrey Modell Foundation, nuestro paciente presentaba tres signos de alarma: dos o más neu- monías dentro de un año, necesidad de antimicrobianos intravenosos y dos o más infecciones profundas; siendo indicación de estudio tener dos o más de un total de diez criterios ${ }^{17}$. Otro antecedente en este caso que apoyó la sospecha de una inmunodeficiencia primaria, fue la consanguineidad de los padres, por lo que el resultado del estudio genético permitirá establecer qué mutación es la responsable de su enfermedad, lo que será muy importante para su tratamiento, pronóstico y consejo genético familiar.

Dentro de los pilares del tratamiento de la EGC a largo plazo se encuentran la profilaxis y la terapia antimicrobiana específica de las infecciones. En un ensayo controlado, se encontró que sin profilaxis antimicrobiana, los pacientes con EGC sufrieron, en promedio, un episodio infeccioso potencialmente mortal cada 10 meses, mientras que los que recibieron profilaxis con cotrimoxazol, la tasa de infección se redujo a un episodio potencialmente mortal cada 40 meses $^{18}$. Cotrimoxazol es el antimicrobiano de elección como profilaxis bacteriana en la EGC, dado su amplio espectro y especial cobertura frente a Nocardia spp. al usarlo en forma diaria. Además, alcanza una buena concentración en las células polimorfonucleares y no afecta la microbiota anaerobia intestinal $^{19}$ (Tabla 1). Es importante monitorizar las reacciones adversas a fármacos y en caso de alergia a las sulfas, se puede usar como alternativa trimetoprim sin sulfametoxazol, penicilinas, cefalosporinas o fluoroquinolonas. En cuanto a la prevención de infecciones por hongos, un estudio multicéntrico demostró que el uso de profilaxis específica disminuyó la incidencia de enfermedad fúngica invasora en pacientes con EGC respecto de quienes no la recibieron ${ }^{20}$. Aspergillus spp. es la primera causa de infección fúngica en la EGC, aunque también se describen otros hongos dematiáceos y no dematiáceos menos frecuentes. Itraconazol ha sido tradicionalmente el azol de elección como profilaxis, sin embargo, debido a la aparición de microorganismos resistentes, intolerancia y baja absorción de la formulación en cápsulas disponible en nuestro país, ha aumentado el uso de voriconazol y posaconazol. La biodisponibilidad del voriconazol es superior a la del itraconazol y confiere un espectro antifúngico más amplio, con una actividad mejorada contra Aspergillus spp. Posaconazol también proporciona una actividad de amplio espectro y la administración oral es más cómoda que la de itraconazol en pediatría ${ }^{19}$ (Tabla 1).

En conclusión, la EGC es una inmunodeficiencia primaria que afecta la fagocitosis provocando infecciones bacterianas y fúngicas recurrentes en quienes la padecen. Un diagnóstico oportuno, en conjunto con la terapia oportuna y el inicio precoz de profilaxis antimicrobiana son fundamentales en la disminución en la tasa de infecciones y mortalidad. 


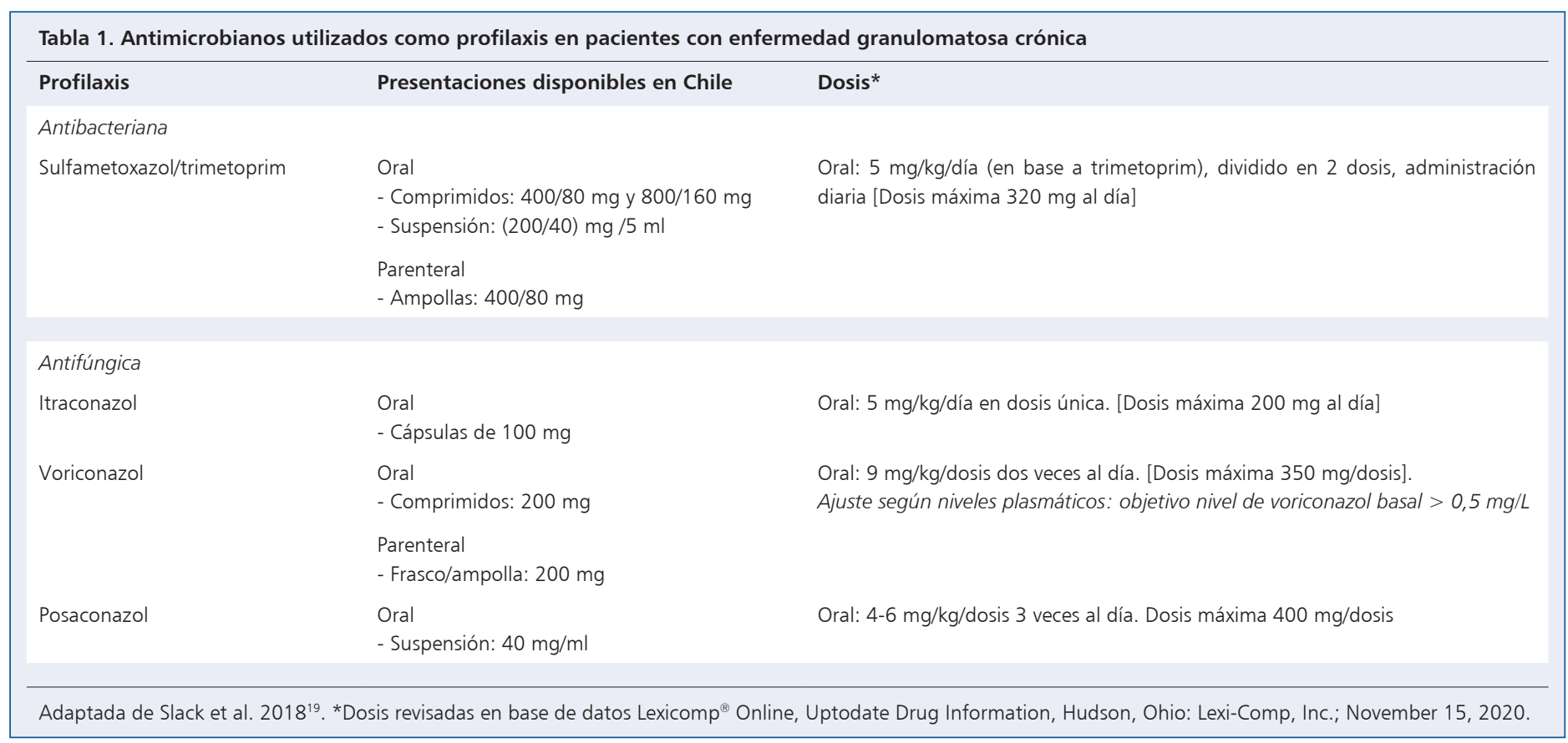

\section{Referencias bibliográficas}

1.- Rider N L, Jameson M B, Creech C B. Chronic granulomatous disease: Epidemiology, pathophysiology, and genetic basis of disease. $\mathrm{J}$ Pediatric Infect Dis Soc. 2018;7(Suppl 1):S2-5. doi: 10.1093/jpids/piy008.

2.- Wolach B, Gavrieli R, de Boer M, van Leeuwen K, Berger-Achituv S, Stauber T, et al. Chronic granulomatous disease: Clinical, functional, molecular, and genetic studies. The Israeli experience with 84 patients. Am J Hematol. 2017;92(1):28-36. doi: 10.1002/ajh.24573.

3.- Suliaman F, Amra N, Sheikh S, Almuhsen S, Alsmadi O. Epidemiology of chronic granulomatous disease of childhood in Eastern Province, Saudi Arabia. Pediatr Asthma Allergy Immunol. 2009;22(1):21-6. doi: 10.1089/ pai.2008.0513.

4.- Segal B H, Leto T L, Gallin J I, Malech H L, Holland S M. Genetic, biochemical and clinical features of chronic granulomatous disease. Medicine (Baltimore). 2000;79(3): 170-200. doi: 10.1097/00005792-20000500000004.

5.- Arnadottir G A, Norddahl G L, Gudmundsdottir S, Agustsdottir A B, Sigurdsson S, Jensson B O, et al. A homozygous loss-of-function mutation leading to CYBC1 deficiency causes chronic granulomatous disease. Nat Commun [Internet]. 2018;9(1):1-9. doi: 10.1038/s41467-01806964-x.

6.- Flannagan R S, Jaumouillé V, Grinstein S. The cell biology of phagocytosis. Annu Rev Pathol Mech Dis. 2012;7:61-98. doi: 10.1146/annurevpathol-011811-132445.

7.- Reeves E, Lu H, Jacobs H, Messina C, Bolsover S, Gabella G, et al. Killing activity of neutrophils is mediated through activation of proteases by K+ flux. Nature. 2002;416(6878):291-7. doi: 10.1038/416291a.

8.- Fuchs T, Abed U, Goosmann C, Hurwitz R, Schulze I, Wahn V, et al. Novel cell death program leads to neutrophil extracellular traps. J Cell Biol. 2007;176(2):231-41. doi: 10.1083/ jcb.200606027.

9.- Galluzzo M L, Hernández C, Dávila M T, Pérez L, Oleastro M, Zelazko M, et al. Clinical and histopathological features and a unique spectrum of organisms significantly associated with chronic granulomatous disease osteomyelitis during childhood. Clin Infect Dis. 2008;46(5):745-9. doi: 10.1086/527446.

10.- Holland S M. Chronic granulomatous disease. Clin Rev Allergy Immunol. 2010;38(1):3-10. doi: 10.1007/s12016-009-8136-z.

11.- Marciano B E, Spalding C, Fitzgerald A, Mann D, Brown T, Osgood S, et al. Common severe infections in chronic granulomatous disease. Clin Infect Dis. 2015;60(8):1176-83. doi: 10.1159/000319855.

12.- van De Veerdonk F L, Smeekens S P, Joosten L A, Kullberg B J, Dinarello C A, van der Meer J W, et al. Reactive oxygen species-independent activation of the IL-1 $\beta$ inflammasome in cells from patients with chronic granulomatous disease. Proc Natl Acad Sci U S A. 2010;107(7):3030-3. doi: 10.1073/ pnas.0914795107.

13.- Salfa I, Cantarutti N, Angelino G, Di Matteo G, Capo V, Farinelli G et al. Serratia marcescens osteomyelitis in a newborn with chronic granulomatous disease. Pediatr Infect Dis J. 2013;32(8):926. doi: 10.1097/ INF.0b013e31828f682a.

14.- van den Berg J M, van Koppen E, Åhlin A, Belohradsky B H, Bernatowska E, Corbeel et al. Chronic granulomatous disease: The European experience. PLoS One. 2009;4(4):1-10. doi: 10.1371/journal. pone. 0005234

15.- Mahlen S D. Serratia infections: From military experiments to current practice. Clin Microbiol Rev. 2011;24(4):755-91. doi: 10.1128/ CMR.00017-11.

16.- Bustamante J, Aksu G, Vogt G, de Beaucoudrey L, Genel F, Chapgier A et al. BCG-osis and tuberculosis in a child with chronic granulomatous disease. J Allergy Clin Immunol. 2007;120(1):32-8. doi: 10.1016/j. jaci.2007.04.034

17.- Modell V, Quinn J, Orange J, Notarangelo LD, Modell F. Primary immunodeficiencies worldwide: an updated overview from the Jeffrey Modell Centers Global Network. Immunol Res. 2016;64(3):736-53. doi: 10.1007/ s12026-016-8784-z.

18.- Gallin J I, Buescher E S, Seligmann BE, Nath J, Gaither T, Katz P. NIH conference. 


\section{Caso Clínico}

Recent advances in chronic granulomatous disease. Ann Intern Med. 1983;99(5):657-74. doi: 10.7326/0003-4819-99-5-657.

19.- Slack M A, Thomsen IP. Prevention of infectious complications in patients with chronic granulomatous disease. J Pediatric Infect Dis Soc. 2018;7(Suppl 1):S25-30. doi: 10.1093/jpids/piy016.

20.- Beauté J, Obenga G, Le Mignot L, Mahlaoui N, Bougnoux ME, Mouy R, et al. Epidemiology and outcome of invasive fungal diseases in patients with chronic granulomatous disease: A multicenter study in France. Pediatr Infect Dis J. 2011;30(1):57-62. doi: 10.1097/ INF.0b013e3181f13b23. 